\title{
Identifying genetic variants underlying medication-induced osteonecrosis of the jaw in cancer and osteoporosis: a case control study
}

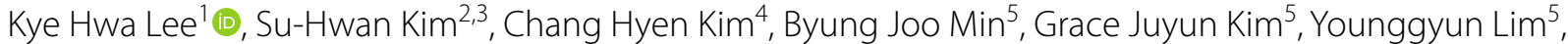 \\ Hun-Sung Kim ${ }^{6}$, Kang-Min Ahn ${ }^{7^{*}}$ and Ju Han Kim ${ }^{1,5^{*}}$
}

\begin{abstract}
Background: Bisphosphonate-induced osteonecrosis of the jaw (BRONJ) presents with a typical pattern of jaw necrosis in patients who have been prescribed bisphosphonates (BPs) and other antiangiogenetic drugs to treat osteoporosis or bone-related complications of cancer.

Methods: This study divided 38 patients with BRONJ into two groups according to the prescribing causes: cancer $(n=13)$ and osteoporosis $(n=25)$, and underwent whole exome sequencing and compared them with normal controls $(n=90)$. To identify candidate genes and variants, we conducted three analyses: a traditional genetic model, gene-wise variant score burden, and rare-variant analysis methods.

Results: The stop-gain mutation (rs117889746) of the PZP gene in the BRONJ cancer group was significantly identified in the additive trend model analysis. In the cancer group, ARIDS, HEBPI, LTBP1, and PLVAP were identified as candidate genes. In the osteoporosis group, VEGFA, DFFA, and FAM193A genes showed a significant association. No significant genes were identified in the rare-variant analysis pipeline. Biologically accountable functions related to BRONJ occurrence-angiogenesis-related signaling (VEGFA and PLVAP genes), TGF- $\beta$ signaling (LTBP1 and PZP genes), heme toxicity (HEBP1) and osteoblast maturation (ARIDS)-were shown in candidate genes.
\end{abstract}

Conclusion: This study showed that the candidate causative genes contributing to the development of BRONJ differ according to the BP dose and background disease.

Keywords: Osteoporosis, Angiogenesis, Pharmacogenomics, Bioinformatics, Cancer biology

\section{Background}

Bisphosphonates (BPs) are antiresorptive agents that are commonly used to treat osteoporosis, multiple myeloma, and metastatic solid tumors [1]. BPs become internalized into osteoclasts via endocytosis and subsequently inhibit their activity [2]. Despite BPs being well known to be effective in preventing osteoporotic fractures and preventing cancer-related side effects in bone, there has

\footnotetext{
*Correspondence: ahnkangmin@ulsan.ac.kr; juhan@snu.ac.kr ${ }^{\dagger}$ Kye Hwa Lee and Su-Hwan Kim-First authors

${ }^{1}$ Center for Precision Medicine, Seoul National University Hospital, Seoul 03082, South Korea

${ }^{7}$ Department of Oral and Maxillofacial Surgery, University of Ulsan College of Medicine, Seoul 05505, South Korea

Full list of author information is available at the end of the article
}

been a sharp decline in BP prescriptions in recent years, from 21.3 million in 2002 to 14.7 million in 2012 in the United States, with increasing reports of diverse rare but serious side effects associated with the use of BPs [3]. Osteonecrosis of the jaw $(\mathrm{ONJ})$ is one of the most-serious and well-known side effects of BPs [4]. ONJ is characterized by jawbone necrosis, which exposes necrotic bones through holes in mucous membranes or the facial skin, traditionally ranging from a few millimeters to a few centimeters $[2,5]$. The reported incidence rate of $\mathrm{ONJ}$ when using BPs to treat cancer has ranged from 0.7 to $10.3 \%$ [6], while its incidence in osteoporosis has not been established [5, 7]. Because other drugs are also known to be risk factors for ONJ, such as other types of antiresorptive drug (denosumab) and antiangiogenics, the 
American Association of Oral and Maxillofacial Surgeons committee recommended to change the nomenclature of ONJ caused by drugs from BRONJ (bisphosphonaterelated osteonecrosis of the jaw) to medication-related osteonecrosis of the jaw (MRONJ) in 2014 [8].

Apart from the well-known direct causes of MRONJ such as dental surgery or gingival infection, the main mechanism underlying the occurrence of MRONJ has not been clearly elucidated [9]. Since ONJ occurs only in specific individuals, multiple studies have been carried out to confirm the genetic background of MRONJ [9-11]. Despite the dearth of genomic studies and their results not being well replicated, these studies have added a deep pathological understanding of and insight into the development of MRONJ, such as the patient's innate immunity, angiogenesis inhibition, osteoclast suppression, and systemic/local inflammations being strong predisposing factors [8]. Some of the candidate genes identified by these studies are TGFb1, MMP2, PPARG, CYP2C8, VEGF, COL1A1, RANK, OPG, OPN, and RBMS3 [12$16]$. However, most of these studies were either candidate-gene studies or genome-wide association studies (GWASs) [17]. Previous whole exome sequencing (WES) studies have found that multiple biological pathway contribute to the occurrence of MRONJ, but no specific contributing genes have been identified [9]. Recently, a study included total 44 multiple myeloma and 17 solid tumor BRONJ patients of European ancestry using WES was identified protective SNPs with significant linkage disequilibrium with SIRT1 and HERC4 genes [15, 18].

In this study we applied case-control methods that are commonly used in genomics research on complex diseases to identify genes exhibiting large variations between BRONJ patients and healthy control subjects. We divided BRONJ patients into two groups depending on whether BPs had been prescribed for cancer and osteoporosis, based on the assumption that the genetic vulnerabilities contributing to the occurrence of BRONJ differ between the long-term accumulation of BPs in osteoporosis and the high-dose toxicity of BPs in cancer.

\section{Methods}

\section{Study design and participants}

We prospectively collected clinical data and blood and saliva samples from 40 patients diagnosed with BRONJ: 30 at Asan Medical Center from May 2013 to November 2015 and 10 at Seoul St. Mary's Hospital from November 2010 to November 2014. All of the patients were clinically evaluated by dentists and were diagnosed as BRONJ according to the guideline from the American Association of Oral and Maxillofacial Surgeons [8]. All patients had been taken BPs or had a history of BPs prescription before ONJ occurs. They had necrotic lesions in maxillar or mandibular bone and no history of administration of radiation therapy at the necrotic bone area. We performed sample size estimation with $70 \%$ detection power, $20 \%$ significance level, MAF in case and MAF in 1-5\% control, respectively, to identify variations contributing to BRONJ. From the calculation, 16-39 and 48-116 samples were required for the case and control respectively. Therefore, in this study, we started the study with 40 cases and 90 health controls. Excluding two patients who were failed DNA extraction, 38 patients were included in the final study. BPs were prescribed for cancer (multiple myeloma or metastatic cancer) in 13 patients and for osteoporosis in 25 patients. The types of BPs taken by the patients were zoledronate $(n=11)$, alendronate $(n=10)$, risedronate $(n=5)$, pamidronate $(n=3)$, ibandronate $(n=1)$, zoledronate/ibandronate $(n=1)$, or unknown $(n=6)$. The most commonly used drug was zoledronate $(61 \%, n=8)$ in the cancer patients and alendronate $(40 \%, n=10)$ in the osteoporosis patients. Before the onset of BRONJ, BPs had been prescribed for $18.1 \pm 13.9$ months (mean \pm SD) in the cancer patients and $56.0 \pm 52.3$ months in the osteoporosis patients. The clinical phenotypes of the patients are listed in Table 1. The control subjects were enrolled for the previous study of "Physicians' Seq Project" [19]. These normal control group was from the previous sequencing study of physicians to evaluate the physicians' expectation and attitude for pharmacogenomics using their own genome. A detailed description was in the previous research paper. The incidence of $\mathrm{BRONJ}$ among $\mathrm{BP}$ recipients was reported as one in 100,000, which is similar to the occurrence of jaw osteonecrosis (ONJ) in the general population $[7,20,21]$. Thus, we considered that those who had osteoporosis but did not develop ONJ within 6 months to a year would not have significantly different genetic characteristics than the general population.

\section{Sequencing data analysis}

The detailed methods of WES, variant calls, quality control and annotation were described in Additional file 1: Materials and Methods. Because the case group comprised subjects with two causes of disease for BP prescriptions that also showed significantly different clinical features, we divided the cases into two subgroups: the BRONJ cancer group (BC, $n=13)$ and the BRONJ osteoporosis group (BO, $n=25$ ) as shown in Additional file 1: Figure S1.

Because BRONJ patients in our study were mixed with high-dose, intravenous bisphosphonate-treated cancer group and low-dose, per-oral bisphosphonate-treated osteoporosis group, we assumed that the underlying mechanism of ONJ occurrence differ between the two groups, so divided patients into two groups according 
Table 1 Clinical characteristics of the study participants

\begin{tabular}{|c|c|c|c|c|}
\hline & BRONJ total cases $(n=38)$ & $\begin{array}{l}\text { BRONJ cancer cases } \\
(n=13)\end{array}$ & $\begin{array}{l}\text { BRONJ osteoporosis cases } \\
(n=25)\end{array}$ & $\begin{array}{l}\text { Normal } \\
\text { controls } \\
(n=90)\end{array}$ \\
\hline Age, years & $79.9 \pm 11.7$ & $60.1 \pm 8.7$ & $76.6 \pm 8.8$ & $41.1 \pm 7.2$ \\
\hline Males & $8(21 \%)$ & $7(53.8 \%)$ & $2(8.0 \%)$ & $47(52.2 \%)$ \\
\hline $\begin{array}{l}\text { Diagnosed condition resulting in } \mathrm{BP} \\
\text { prescription }\end{array}$ & & & & - \\
\hline Cancer & $13(34.2 \%)$ & $13(100 \%)$ & $0(0 \%)$ & \\
\hline Breast cancer & $6(46.2 \%)$ & $6(46.2 \%)$ & - & \\
\hline Multiple myeloma & $5(38.5 \%)$ & $5(38.5 \%)$ & - & \\
\hline Prostate cancer & $2(15.4 \%)$ & $2(15.4 \%)$ & - & \\
\hline Osteoporosis & $25(65.7 \%)$ & - & $25(100 \%)$ & \\
\hline $\mathrm{BP}$ & & & & - \\
\hline Zoledronate & $11(29.0 \%)$ & $8(61.5 \%)$ & $3(12.0 \%)$ & \\
\hline Alendronate & $10(26.0 \%)$ & - & $10(40.0 \%)$ & \\
\hline Residronate & $5(13.2 \%)$ & - & $5(20.0 \%)$ & \\
\hline Pamidronate & $3(7.9 \%)$ & $3(23.1 \%)$ & - & \\
\hline Ibandronate & $2(5.3 \%)$ & - & $2(8.0 \%)$ & \\
\hline Zoledronate/ibandronate & $1(2.6 \%)$ & $1(7.7 \%)$ & - & \\
\hline Unknown & $6(15.8 \%)$ & $1(7.7 \%)$ & $5(20.0 \%)$ & \\
\hline $\begin{array}{l}\text { Duration of BP treatment before BRONJ } \\
\text { occurrence, months }\end{array}$ & $42.7 \pm 46.3$ & $18.1 \pm 13.9$ & $56.0 \pm 52.3$ & - \\
\hline
\end{tabular}

Data are mean \pm SD or $n(\%)$ values

their background disease and BPs dosage and compared each group with the normal control $(n=90)$. To identify candidate genes and variants associated with BRONJ for each group, we used three analytical methods. Significant genes/variants identified from the three methods were classified using gene set enrichment analysis and we reviewed literatures to evaluate fundamental pathophysiology of BRONJ. The three analysis pipeline is demonstrated in Fig. 1.

We applied three analysis methods to identify BRONJassociated variants and genes. First, three different traditional genetic testing models were used to compare variant frequencies between the cases and controls. This method has a solid statistical basis but also a low statistical power in detecting variants exhibiting mild effects and low frequencies. Second, collapsing analysis with the gene as a unit of measurement was used to assess the damaging effects of all deleterious variants in the genes. This is similar to other burden-based tests that collapse all rare and/or damaging variants within a region into a single value, but our method yielded a score from 0 to 1 that reflected the variability of the variant distribution in a gene. The third analysis method was the rare-variant association test using a multiple regression model while adjusting covariates in order to identify rare variants that affect the phenotype.

\section{Case-control test of variant frequencies}

We performed a case-control association analysis with the variant-allele frequencies (AFs) using the SnpSift CaseControl tool for three different genetic models [22] for each $\mathrm{BC}$ and each $\mathrm{BO}$ versus controls. The statistical tests used were the Cochran-Armitage test for trends and Fisher's exact test (FET) for the dominant and recessive models. For the trend model, we applied weights of 0,1 , and 2 for the reference homozygous, alternative heterozygous, and alternative homozygous variants, respectively. To identify SNPs and INDELs that significantly impacted the function of the corresponding protein, the loss of function (LoF) variants defined as follows were used for further analysis: stop gain/loss, coding INDELs, splice-site acceptors, and splice-site donors. We also included variants predicted as damaging according to their SIFT [23] score and a CADD [24] score of $>20$.

\section{Gene-based collapsing analysis}

In order to measure the degree of damage of a specific gene, we used a previous algorithm to convert the number of a mutations in a gene into a gene score $[25,26]$. The gene score (a gene deleteriousness score) quantified the impact of damage of a gene, and was defined as the geometric mean of the SIFT scores for the multitude of deleterious variants in a gene. The gene score represents an estimate of the aggregate impact of all deleterious 


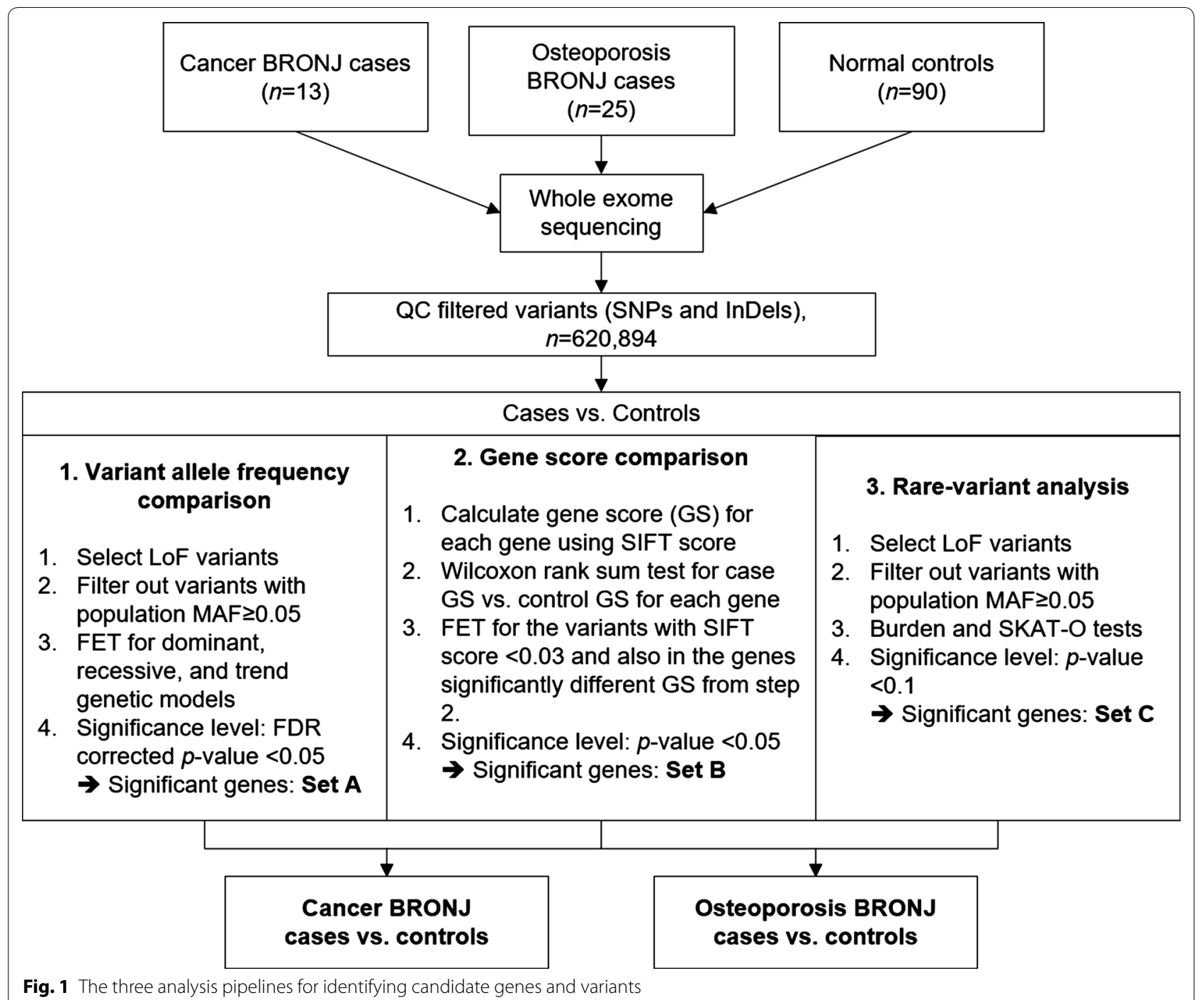

variants in the genes. Since the SIFT score ranges from 0 to 1 and is lower for deleterious variants, a lower gene score indicates greater damage to the function of the gene at the protein level. We identified genes with different gene scores between cases and controls using Wilcoxon rank-sum test. For further analyzing which variants would be responsible for the deleteriousness of a specific gene, we used FET to compare the variant frequencies with all SIFT-mapped variants.

Rare-variant association analysis

To evaluate the effect of rare functional variants that were only observed as phenotype manifestations in one or two patients, we applied the sequence kernel association test (SKAT) for rare LoF variants [27]. The threshold for rare variants was a $\mathrm{MAF}<0.5 \%$ in the Asian population of phase I of the 1000 Genomes [28] and the ExAC project
[29] from the SnpSift annotation. Functionally damaging variants included previously defined LoF variants. Sex and age were used as covariates, and the threshold for significance was a $p$ value of 0.1 after correcting for multiple-tests bias. We used the freely available $\mathrm{R}$ package to apply the SKAT with a small-sample option. Statistical analyses including multiple-tests correction were implemented using custom scripts in R (v3.1.5, R Foundation for Statistical Computing, Vienna, Austria) [30].

\section{Results}

Variant frequency analysis

To identify genetic variants associated with BRONJ, we performed statistical tests with three genetic modelsdominant, recessive, and Cochran-Armitage trend models-for the two case groups (13 BC and $25 \mathrm{BO}$ ) versus 90 healthy controls. Among 519,375 SNPs/INDELs and 
23,420 genes, we extracted 2646 SNPs/INDELs and 2327 genes with LoF variants and performed statistical tests for the BC. LoF variants include stop gain/loss, coding INDELs, splice-site acceptors, splice-site donors, and also variants that were predicted as damaging according to both the SIFT [23] and CADD [24] scores. For BO there were 3684 SNPs/INDELs and 3101 genes with LoF variants out of the total of 693,497 SNPs/INDELs and 23,534 genes. To exclude population-wise major variants that were likely to be benign, variants with a MAF $>0.5 \%$ in the Asian population from phase I of the 1000 Genomes Project [28] and the ExAC [29] were filtered out. This left 343 variants in 335 genes for BC and 367 variants in 357 genes for the BO. Using SnpSift case-control analysis, we performed tests for the three genetics models for all of these LoF variants: dominant, recessive, and additive trend models. After correcting for multiple-tests bias, a stop-gain variant in $P Z P$ for the $\mathrm{BC}$ versus controls was identified in the trend model that was associated with a higher BRONJ risk (Table 2). There was no significant variant in $\mathrm{BO}$ versus controls.

\section{Gene-score comparisons}

After excluding probably benign hypervariable genes including transcription factors (Additional file 1: Results), we performed a clustering analysis using the DAVID Functional Annotation Clustering Tool [31] to evaluate common biological functions shared in these gene sets on 232 and 564 genes for the $\mathrm{BC}$ and $\mathrm{BO}$, respectively. For functional terms mapped to the pathway databases, there were eight clusters for $\mathrm{BC}$ versus controls and seven for $\mathrm{BO}$ versus controls with a significance of $p<0.05$. The enrichment score of each group and statistical test results for the $\mathrm{BC}$ and $\mathrm{BO}$ are listed in Additional file 1: Tables S1, S2, respectively. All of the annotated genes are presented in Additional file 1: Table S3.

To identify contributing variants for cases with lower gene scores, we performed a FET of the AF of all variants with SIFT scores of $<0.3$ among the functionally damaging variants in the case versus control groups. Functionally damaging variants are defined in Additional file 1: Figure S2. At this step there were 10,087 and 12,952 variants for $\mathrm{BC}$ and $\mathrm{BO}$, respectively, among which the number of intersection of variants having significantly different gene scores were 878 and 1647, respectively. Then 161 variants in the $\mathrm{BC}$ and 444 variants in the $\mathrm{BO}$ with higher AF for cases than controls $(p<0.05$, odds ratio $>1$ ) were filtered in. To filter out population major variants and false-positive results, we excluded variants with lower AFs in 90 healthy controls than in the ExAC Asian or the 1000 Genomes Project Asian population (12 variants in the $\mathrm{BC}$ and 15 variants in the $\mathrm{BO}$ ). After removing the variants with a zero allele count in the control group, six and five variants were identified and validated using IGV viewer for the $\mathrm{BC}$ and $\mathrm{BO}$, respectively. From the gene-score analysis pipeline, we identified ARID2, CDC27, HEBP1, LTBP1, PLVAP, and TNRC18 in the BC, and CDC27, DFFA, FAM193A, TNRC18, and $V E G F A$ in the $\mathrm{BO}$ (Tables 3,4$)$. To identify the clusters of patients for these genes, we drew the heat map for the gene score (Fig. 2).

Hierarchical clustering analyses revealed the exclusive pattern of genes related to angiogenesis (PLVAP) and genes related to TGF- $\beta$ signaling (LTBP1 and PZP) in the BC. Despite TNRC18 and CDC27 showed significant differences in both $\mathrm{BC}$ and $\mathrm{BO}$ but those genes do not have definite explainable functions underlying BRONJ. In $\mathrm{BO}$, $D F F A$ and FAM193A genes also significantly enriched but do not have any known underlying function related to ONJ. Otherwise, VEFGA gene in BO and PLVAP, HEBP1, and $L T B P 1$ gene in $B C$ could be applied to explain the BRONJ mechanism. ARID2 gene showed damaged score in eight patients, and also known as cancer-related gene, but hardly having a pathophysiological relationship to BRONJ.

\section{Rare-variant association analysis}

We performed the SKAT-O and burden test for BC versus controls and for $\mathrm{BO}$ versus controls including $\mathrm{LoF}$ variants with a $\mathrm{MAF}<0.05 \%$ in the Asian population of the ExAC project and the 1000 Genomes Project phaseI data. The total number of genes with rare LoF variants was 112 in the $\mathrm{BC}$ and 121 in the BO. There were no genes consistent with the FDR-corrected probability criterion of $p<0.05$ in either the $\mathrm{BC}$ or $\mathrm{BO}$. For $\mathrm{BO}$ versus controls, 60 genes were significantly associated with the risk of BRONJ in the SKAT-O analysis without multiple testing correction, and there were none

Table 2 Results of the variant-allele-frequency association analysis in BC

\begin{tabular}{|c|c|c|c|c|c|c|c|c|c|c|c|}
\hline \multirow[t]{3}{*}{ Chr } & \multirow[t]{3}{*}{ POS } & \multirow[t]{3}{*}{ rsID } & \multirow[t]{3}{*}{ Ref/Alt } & \multirow[t]{3}{*}{ Gene } & \multirow[t]{3}{*}{ Function } & \multirow[t]{3}{*}{ SIFT/CADD } & \multicolumn{2}{|c|}{ Genotype counts } & \multicolumn{2}{|c|}{ Allele frequency } & \multirow[t]{3}{*}{$p$ value } \\
\hline & & & & & & & Case $(n=13)$ & Control $(n=90)$ & $1 \mathrm{KP}$ ASN & ExAC ASN & \\
\hline & & & & & & & GG/GA/AA & GG/GA/AA & & & \\
\hline chr12 & $9,333,626$ & rs117889746 & $\mathrm{G} / \mathrm{A}$ & $P Z P^{a}$ & Stop gain & $0.3 / 36$ & $11 / 1 / 1$ & 70/20/0 & 0.095 & 0.083 & 0.001 \\
\hline
\end{tabular}

a PZP, PZP, alpha-2-macroglobulin like 


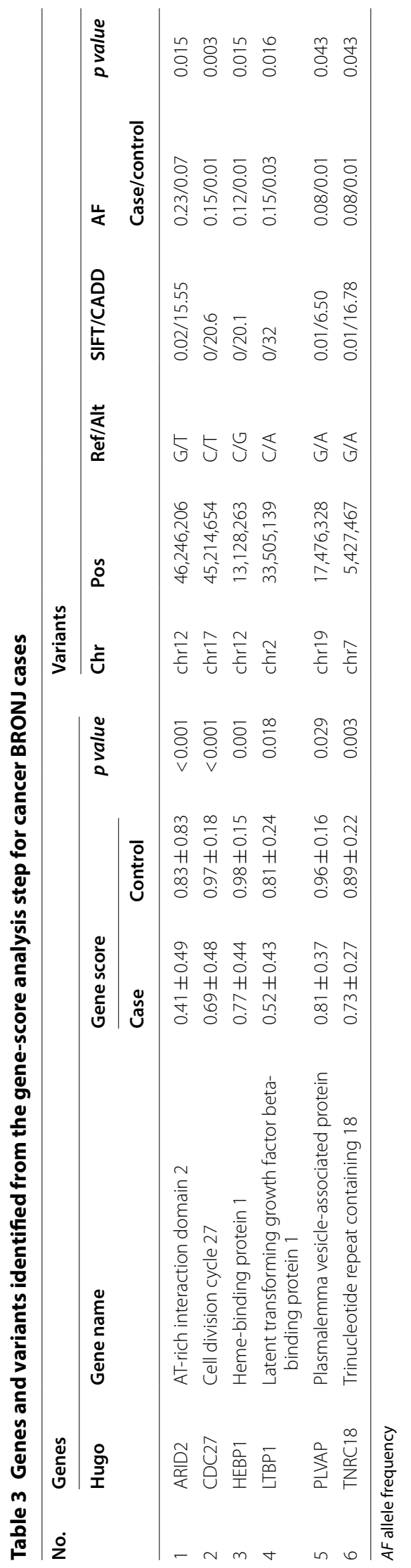




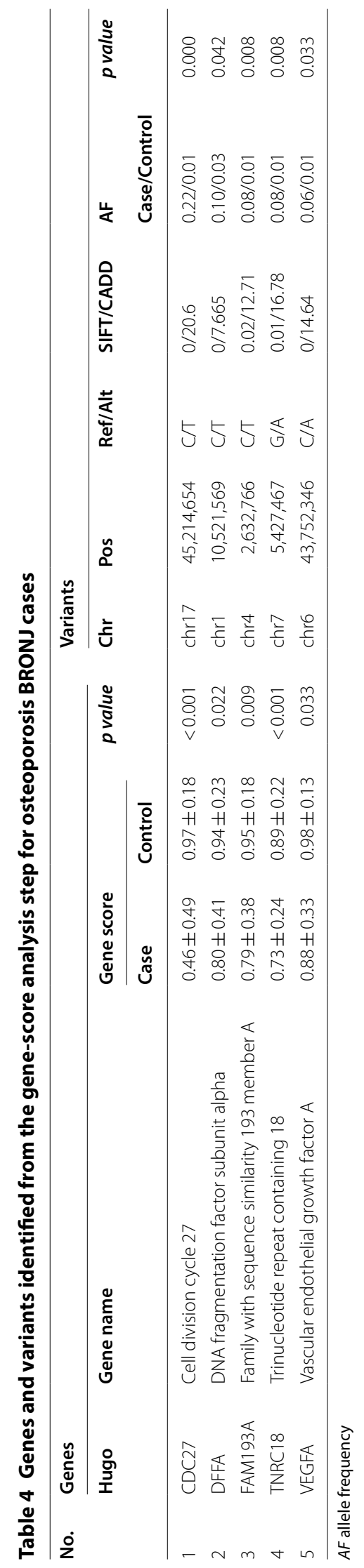




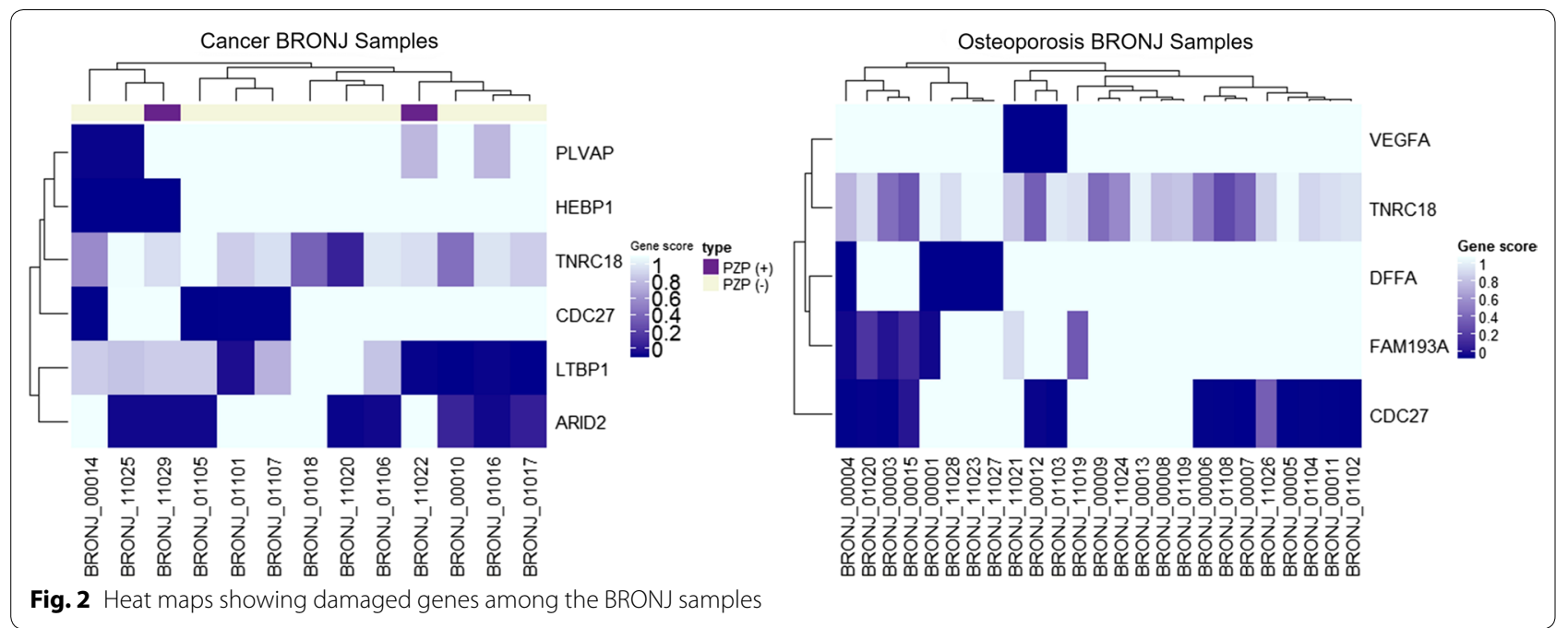

in the burden test. The biological process GO term for proteolysis (GO:0006508) was significantly enriched $(p=0.02)$, with an enrichment score of 1.21 , including six genes (ENDOU, MMP8, CPNE1, TMPRSS7, KLK10, and PRSS42) from the gene set enrichment analysis. There were no genes associated an increased or decreased risk of $\mathrm{BRONJ}$ occurrence in the $\mathrm{BC}$.

\section{Discussion}

This study evaluated the genes associated with the predisposition to develop BRONJ by comparing patients according to the reason for them being prescribed BPs (cancer or osteoporosis) using WES. The genes identified in our study-LTBP1, PZP, ARID2, and HEBP1 in osteoporosis BRON patients-clearly support the previous evidence that angiogenesis, osteoclast activity, bone remodeling, and immune responses are critical underlying mechanisms. In osteoporosis BRONJ group, we identified the $V E G F A$ gene which is known to play a significant role in angiogenesis was also found in previous studies to be associated with the risk of ONJ [11]. We also identified a novel gene associated with the risk of BRONJ that is involved in angiogenesis in patients of cancer BRONJ, PLVAP, which is the VEGFA downstream signaling target involved in the structure of the diaphragm and functions in vascular fenestrations [32]. Other genes identified in cancer group also have ONJ related functions: the $P Z P$ and $L T B P 1$ genes are involved in TGF- $\beta$ signaling (which plays an important role in bone remodeling and tissue repair), the HEBP1 gene is involved in heme pathophysiology, and the ARID2 gene is involved in osteoblast differentiation. These findings suggest that exposure to high-dose BPs in patients with cancer with dysfunctional genes with various underlying pathophysiologies of ONJ increase the risk of BRONJ occurrence. On the other hand, in the osteoporosis BRONJ with a relatively long-term exposure to BPs $(42.7 \pm 46.3$ months), there were no more candidate genes to explain the pathophysiology besides VEGFA.

Previous studies investigated to identify the contributing genetic profile of BRONJ development include two GWAS studies [10, 33], eleven candidate gene studies [11-15, 17, 34-38], and two WES studies [9, 18]. Most of these studies were case-control studies involving less than one hundred single-race patients for BRONJ in cancer patients. The candidate genes and SNPs identified through these studies varied and rarely replicated in another. The pathogenesis of BRONJ is not clearly defined, however, some hypothesis has been suggested $[39,40]$. First, BPs strongly inhibits the activity of osteoclasts and induced apoptosis of osteoclasts. This reduces both bone absorption and formation. Second, BPs inhibit angiogenesis reducing blood vessel distribution in the bone along with inhibiting endothelial growth factor, which interferes with bone remodeling and wound healing in the jaw bone. Lastly, owing to the strong affinity of BPs to hydroxyapatite and long half-life leads to extreme suppression of bone turnover as well as wound healing. As the results of our study suggested as well, damaged genes involved in different but still diverse underlying mechanisms might contribute to the development of BRONJ with diverse mechanisms especially in patients with distinct underlying diseases with very different dosage and potency of BPs.

We used three analysis pipelines to identify candidate genes in order to minimize the false negative caused by various effects of causative genes and genetic variations contributing to BRONJ. The statistical technique using 
the traditional genetic model showed that the stop-gain mutation (rs117889746) in exon 15 of the PZP gene was significantly associated with the occurrence of BRONJ in 2 of the 13 cancer patients ( 1 homozygous and 1 heterozygous). These two patients developed ONJ after receiving Zoledronate injections for 10 months and 24 months after dental procedures such as extraction and implant removal. The $P Z P$ protein as a pan protease inhibitor is involved in the main mechanisms underlying the development of BRONJ: bone formation and inflammation. PZP protein is similar to $\alpha 2$-macroglobulin and has a high affinity with TGF- $\beta 1$ and TGF- $\beta 2$. Binding by $P Z P$ prevents TGFs from binding to cell-surface receptors, which in turn can eliminate TGF- $\beta$ according to the morphological changes in PZP, and also act as a carrier [41]. TGF- $\beta$ promotes tissue repair by enhancing the transcription of type I collagen, which is the main component of the extracellular matrix (ECM). Previous studies have shown that the expression of TGF- $\beta$ is significantly reduced in specimens obtained from patients with nontraumatic osteonecrosis of the femoral head [42]. This is consistent with previous immunohistochemistry studies of the TGF- $\beta 1$ signaling molecule in BRONJ patients showing significantly reduced TGF- $\beta 1$ and Smad- $2 / 3$ in BRONJ patients compared to osteoradionecrosis patients [43]. Previous studies have shown that TGF- $\beta$ promotes bone resorption of the mouse calvariae bone resorption at low doses and does not promote the resorption of the long bones at high doses [44]. Therefore, the results of our study suggest that the TGF- $\beta$ signaling involved in ECM repair is related to the occurrence of BRONJ.

Our utilization of a gene-score analysis pipeline allowed us to identify more candidate genes than when using traditional genetic models. Excluding genes without known specific functions (CDC27 and TNRC18), ARID2, HEBP1, LTBP1, and PLVAP were the only significant differences in the cancer group revealed by the genescore methodology, while DFFA, FAM193A, and VEGFA were the only significant differences in the osteoporosis group. In particular, the VEGFA gene, which differed significantly in the osteoporosis BRONJ, is a member of previous well known risk gene families in ONJ [12], VEGF which is a growth factor that plays an important role in angiogenesis, vasculogenesis, and epithelial cell growth [42]. It has long been known that VEFG plays an important role in bone formation and repair [41], and there has also been a GWAS supporting the hypothesis that the impairment of angiogenesis in the tissue surrounding unnecrotized tissue would be involved in the development of BRONJ [11]. In addition, the PLVAP gene, which is involved in the structure of the diaphragm and vascular fenestrations identified in the cancer group, may be a downstream target of $V E G F$ signaling, and is also an important factor in angiogenesis. Otherwise, the LTBP1 gene that is related to osteoclast activity, which is involved in bone remodeling and the development of BRONJ, has been newly identified in this study. This gene has been shown to release the active form of TGF- $\beta 1$ in the ECM [45], and it plays an important role in osteogenesis and bone resorption. Thus, dysfunction of the LTBP1 gene might also be implicated in the development of BRONJ. The HEBP1 gene identified in three BRONJ cancer patients is very interesting as well. This gene codes Heme Binding Protein 1 (HBP1), and heme is a complex of iron and tetrapyrrole protoporphyrin IX, which is in the prosthetic group of hemoproteins that play a key role in oxygen binding and the transportation of compounds such as hemoglobin and myoglobin [46]. An elevated concentration of free heme can induce pro-oxidant, proinflammatory, and cytotoxic effects that affect different cell types. Heme toxicity plays a major role in the pathogenesis of hemolytic disorders such as sickle-cell disease. Only a few studies have investigated the effects of dysfunction of the $H E B P 1$ gene on HBP production and metabolism, but heme toxicity and BRONJ present with very similar symptoms, and so further studies are needed into this association.

\section{Conclusions}

We identified genes enriched significantly different between cancer and osteoporosis BRONJ group. Despite the small number of patients, the genes related to the pathophysiology in BRONJ occurrence were more enriched in the cancer group than osteoporosis. The limitations of our study are that we could not prove candidate genes and mutations derived from the study through additional testing besides the single ethnicity of participants (all East Asian). The results of this study need to be verified in future replication studies. It is well known that high doses of BPs increase the risk of BRONJ. Our results suggest that BRONJ may occur more easily in patients with impaired function of angiogenesis, osteoclast activity, and tissue repair with high dose BPs. If additional studies are conducted on a more sufficient patient population, the conclusions of this study should be supported.

\section{Supplementary information}

Supplementary information accompanies this paper at https://doi. org/10.1186/s12967-019-2129-3.

Additional file 1. Suuplementary information.

\section{Acknowledgements}

Not applicable. 


\section{Authors' contributions}

KMA and JHK designed the study and KHL and SHK wrote the draft manuscript and KHL and GJK draw figures and tables. KHL, SHK, CHK, KMA, HSK and JHK wrote the main manuscript text and BJM, GJK and YL prepared and analysis the exome data. All authors reviewed the manuscript. All authors read and approved the final manuscript.

\section{Funding}

This work was supported by the Education and Research Encouragement Fund of Seoul National University Hospital and the National Research Foundation of Korea (NRF) funded by the Ministry of Education (NRF2018R1D1A1A02086109). There are no conflicts of interest to declare.

\section{Availability of data and materials}

The datasets generated and/or analysed during the current study are not publicly available due to the consent limitation of the study participants but are available from the corresponding author on reasonable request.

\section{Ethics approval and consent to participate}

This research involving human subjects and their genomic data was approved by the institutional review boards of Asan Medical Center and Seoul St. Mary's Hospital (IRB nos. 2014-1216 and KC15TIMI0055, respectively). The control subjects were recruited and approved by the institutional review boards of Seoul National University Hospital (IRB no. 1507-093-689). Written informed consent was obtained from each subject prior to their participation. We confirmed the checklist according to the STROBE guidelines and satisfied all items.

\section{Consent for publication}

Not applicable.

\section{Competing interests}

The authors declare that they have no competing interests.

\section{Author details}

${ }^{1}$ Center for Precision Medicine, Seoul National University Hospital, Seoul 03082, South Korea. ${ }^{2}$ Department of Periodontics, Asan Medical Center, Seoul 05505, South Korea. ${ }^{3}$ Department of Dentistry, University of Ulsan College of Medicine, Seoul 05505, South Korea. ${ }^{4}$ Department of Oral and Maxillofacial Surgery, Seoul St. Mary's Hospital, College of Medicine, The Catholic University of Korea, Seoul 06591, South Korea. ${ }^{5}$ Division of Biomedical Informatics, Seoul National University Biomedical Informatics (SNUBI) and Systems Biomedical Informatics Research Center, Seoul National University College of Medicine, Seoul 03080, South Korea. ${ }^{6}$ Department of Medical Informatics, College of Medicine, The Catholic University of Korea, Seoul 06591, Republic of Korea. ${ }^{7}$ Department of Oral and Maxillofacial Surgery, University of Ulsan College of Medicine, Seoul 05505, South Korea.

Received: 24 August 2019 Accepted: 5 November 2019

Published online: 20 November 2019

\section{References}

1. Favus MJ. Bisphosphonates for osteoporosis. N Engl J Med. 2010;363:2027-35.

2. Fung PL, et al. Pharmacogenetics of bisphosphonate-associated osteonecrosis of the jaw. Oral Maxillofac Surg Clin N Am. 2015:27(4):537-46.

3. Wysowski DK, Greene P. Trends in osteoporosis treatment with oral and intravenous bisphosphonates in the United States, 2002-2012. Bone. 2015;57(2):423-8

4. Woo S-B, Hellstein JW, Kalmar JR. Systematic review: bisphosphonates and osteonecrosis of the jaws. Ann Intern Med. 2006;144(10):753.

5. Khosla S, et al. Bisphosphonate-associated osteonecrosis of the jaw: report of a task force of the American Society for Bone and Mineral Research. J Bone Miner Res. 2007;22(10):1479-91.

6. Rugani $\mathrm{P}$, et al. Prevalence of bisphosphonate-associated osteonecrosis of the jaw after intravenous zoledronate infusions in patients with early breast cancer. Clin Oral Investig. 2014;18(2):401-7.

7. Reid IR, Cornish J. Epidemiology and pathogenesis of osteonecrosis of the jaw. Nat Rev Rheumatol. 2012;8(2):90-6.
8. Ruggiero SL, et al. American Association of oral and maxillofacial surgeons position paper on medication-related osteonecrosis of the jaw-2014 update. J Oral Maxillofac Surg. 2014;72(10):1938-56.

9. Kim JH, et al. Genetic investigation of bisphosphonate-related osteonecrosis of jaw (BRONJ) via whole exome sequencing and bioinformatics. PLOS ONE. 2015;10(2):e0118084.

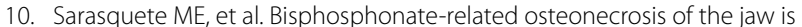
associated with polymorphisms of the cytochrome P450 CYP2C8 in multiple myeloma: a genome-wide single nucleotide polymorphism analysis. Blood. 2008;112(7):2709-12.

11. Choi H, Lee J, Lee J-H, Kim J-H. Genetic association between VEGF polymorphisms and BRONJ in the Korean population. Oral Dis. 2015;21(7):866-71.

12. Arduino PG, et al. Vascular endothelial growth factor genetic polymorphisms and haplotypes in female patients with bisphosphonate-related osteonecrosis of the jaws. J Oral Pathol Med. 2011;40(6):510-5.

13. Balla B, et al. New approach to analyze genetic and clinical data in bisphosphonate-induced osteonecrosis of the jaw. Oral Dis. 2012;18(6):580-5

14. Katz J, et al. Genetic polymorphisms and other risk factors associated with bisphosphonate induced osteonecrosis of the jaw. Int J Oral Maxillofac Surg. 2011;40(6):605-11.

15. Di Martino MT, et al. A peroxisome proliferator-activated receptor gamma (PPARG) polymorphism is associated with zoledronic acid-related osteonecrosis of the jaw in multiple myeloma patients: analysis by DMET microarray profiling. Br J Haematol. 2011;154:529-33. https://doi. org/10.1111/j.1365-2141.2011.08622.x

16. Yang $\mathrm{G}$, et al. Pharmacogenomics of osteonecrosis of the jaw. Bone. 2019;124:75-82. https://doi.org/10.1016/j.bone.2019.04.010.

17. Marini F, et al. Pharmacogenetics of bisphosphonate-associated osteonecrosis of the jaw. Front Biosci (Elite Ed). 2011;3:364-70.

18. Yang G, et al. SIRT1/HERC4 locus associated with bisphosphonateinduced osteonecrosis of the jaw: an exome-wide association analysis. J Bone Miner Res. 2018;33(1):91-8.

19. Lee KH, Min BJ, Kim JH. Personal genome testing on physicians improves attitudes on pharmacogenomic approaches. PLoS ONE. 2019;14(3):e0213860.

20. Khan AA, Rios LP, Sandor GK, Khan N, Peters E, Rahman MO, et al. Bisphosphonate-associated osteonecrosis of the jaw in Ontario: a survey of oral and maxillofacial surgeons. J Rheumatol. 2011;38:1396-402.

21. Assael LA. Oral bisphosphonates as a cause of bisphosphonate-related osteonecrosis of the jaws: clinical findings, assessment of risks, and preventive strategies. J Oral Maxillofac Surg. 2009;67:35-43.

22. Cingolani $P$, et al. Using Drosophila melanogaster as a model for genotoxic chemical mutational studies with a new program, SnpSift. Front Genet. 2012:15(3):35.

23. Ng Pauline $\mathrm{C}$, Henikoff Steven. SIFT: predicting amino acid changes that affect protein function. Nucleic Acids Res. 2003;31(13):3812-4.

24. Rentzsch $P$, et al. CADD: predicting the deleteriousness of variants throughout the human genome. Nucleic Acids Res. 2018;47(D1):D886-94

25. Lee $\mathrm{KH}$, et al. Genome sequence variability predicts drug precautions and withdrawals from the market. PLoS ONE. 2016;11(9):e0162135.

26. Seo H, et al. Deleterious genetic variants in ciliopathy genes increase risk of ritodrine-induced cardiac and pulmonary side effects. BMC Med Genomics. 2018;11(1):4

27. Wu MC, et al. Rare-variant association testing for sequencing data with the sequence kernel association test. Am J Hum Genet. 2011;89(1):82-93.

28. Genomes Project Consortium, Auton A, Brooks LD. A global reference for human genetic variation. Nature. 2015;526:68-74.

29. Lek M, Karczewski KJ, Minikel EV, Samocha KE, Banks E, Fennell T, et al. Analysis of protein-coding genetic variation in 60,706 humans. Nature. 2015;30:285-91.

30. R Core Team. R: a language and environment for statistical computing. Vienna: R Foundation for Statistical Computing. https://www.R-proje ct.org/.

31. Huang DW, et al. The DAVID Gene Functional Classification Tool: a novel biological module-centric algorithm to functionally analyze large gene lists. Genome Biol. 2007;8(9):R183.

32. Strickland LA, Jubb AM, Hongo JA, Zhong F, Burwick J, Fu L, Frantz $\mathrm{GD}$, Koeppen H. Plasmalemmal vesicle-associated protein (PLVAP) is 
expressed by tumour endothelium and is upregulated by vascular endothelial growth factor-A (VEGF). J Pathol. 2005;206(4):466-75.

33. Nicoletti P, et al. Genomewide pharmacogenetics of bisphosphonateinduced osteonecrosis of the jaw: the role of RBMS3. Oncologist. 2012;17(2):279-87.

34. Stockmann P, et al. Major histocompatibility complex class II polymorphisms are associated with the development of anti-resorptive agentinduced osteonecrosis of the jaw. J Craniomaxillofac Surg. 2013;41:71-5.

35. English BC, et al. A SNP in CYP2C8 is not associated with the development of bisphosphonate-related osteonecrosis of the jaw in men with castrate-resistant prostate cancer. Ther Clin Risk Manag. 2010;6:579-83.

36. Kastritis $\mathrm{E}$, et al. Genetic factors related with early onset of osteonecrosis of the jaw in patients with multiple myeloma under zoledronic acid therapy. Leuk Lymphoma. 2017;58:2304-9.

37. La Ferla F, et al. An aromatase polymorphism (g.132810C $>$ T) predicts risk of bisphosphonate-related osteonecrosis of the jaw. Biomark Med. 2012;6:201-9.

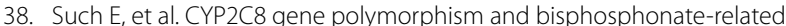
osteonecrosis of the jaw in patients with multiple myeloma. Haematologica. 2011:96(10):1557-9.

39. Tetsuro Ikebe. Pathophysiology of BRONJ: drug-related osteoclastic disease of the jaw. Oral Sci Int. 2013;10(1):1-8.

40. Russell, et al. Bisphosphonates: pharmacology, mechanisms of action and clinical uses. Osteoporos Int. 1999;9(8):S66-80.
41. Keyt BA, et al. The carboxyl-terminal domain (111165) of vascular endothelial growth factor is critical for its mitogenic potency. J Biol Chem. 1996;271(13):7788-95.

42. Uniprot consortium T, Bateman A, Martin MJ, O'Donovan C, Magrane M, Alpi E, Antunes R, et al. UniProt: the universal protein knowledgebase. Nucleic Acids Res. 2017;45(D1):D158-69.

43. Philip A, Bostedt L, Stigbrand T, O'Connor-Mccourt MD. Binding of transforming growth factor- $\beta$ (TGF- $\beta$ ) to pregnancy zone protein (PZP). Eur J Biochem. 1994:221(2):687-93.

44. Pfeilschifter J, Seyedin SM, Mundy GR. Transforming growth factor beta inhibits bone resorption in fetal rat long bone cultures. J Clin Invest. 1998;82(2):680-5.

45. Åström P. Regulatory mechanisms mediating matrix metalloproteinase-8 effects in oral tissue repair and tongue cancer. University of Oulu. 2014.

46. Immenschuh S, Vijayan V, Janciauskiene S, Gueler F. Heme as a target for therapeutic interventions. Front Pharmacol. 2017;8:146.

\section{Publisher's Note}

Springer Nature remains neutral with regard to jurisdictional claims in published maps and institutional affiliations.
Ready to submit your research? Choose BMC and benefit from:

- fast, convenient online submission

- thorough peer review by experienced researchers in your field

- rapid publication on acceptance

- support for research data, including large and complex data types

- gold Open Access which fosters wider collaboration and increased citations

- maximum visibility for your research: over $100 \mathrm{M}$ website views per year

At BMC, research is always in progress.

Learn more biomedcentral.com/submissions 